\title{
Intelligent Systems for Volumetric Feature Recognition from CAD Mesh Models
}

\author{
Vaibhav Hase ${ }^{1[0000-0002-5999-5175]}$, Yogesh Bhalerao ${ }^{2[0000-0002-0743-8633]}$, Saurabh \\ Verma $^{3[0000-0002-2093-0833]}$,G.J. Vikhe ${ }^{4 \text { [0000-0001-6114-5877] }}$ \\ ${ }^{1}$ Research scholar, SPPU, Department of Mechanical Engineering, AVCOE, Sangamner, India \\ ${ }^{2}$ Professor in Mechanical Engineering, MIT Academy of Engineering, Alandi, Pune, India \\ ${ }^{3}$ Centre for Computational Technologies, Pune, India \\ ${ }^{4}$ SPPU, Department of Mechanical Engineering, AVCOE, Sangamner, India \\ hase.vaibhav@avcoe.org
}

\begin{abstract}
This paper presents an intelligent technique to recognize the volumetric features from CAD mesh models based on hybrid mesh segmentation. The hybrid approach is an intelligent blending of facet-based, vertex based, rulebased, and machine learning based techniques. Comparing with existing stateof-the-art approaches, the proposed approach does not depend on attributes like curvature, minimum feature dimension, number of clusters, number of cutting planes, the orientation of model and thickness of the slice to extract volumetric features. The intelligent threshold prediction makes hybrid mesh segmentation automatic. The proposed technique automatically extracts volumetric features like blends and intersecting holes along with their geometric parameters. The proposed approach has been extensively tested on various benchmark test cases. The proposed approach outperforms the existing techniques favorably and found to be robust and consistent with coverage of more than $95 \%$ in addressing volumetric features
\end{abstract}

Keywords: CAD mesh model, Hybrid mesh segmentation, Volumetric feature recognition, Threshold Prediction.

\section{Introduction}

Volumetric features are ubiquitous in mechanical engineering applications from design to manufacturing cycle. In many mechanical engineering parts, blends, holes constitute a significant percentage of features. Recognizing volumetric features in CAD mesh models is vital in applications such as mesh simplification, design, manufacturing, and FEA.

Mesh models constructed from 3D scan data are called scan derived mesh and those generated from B-rep models using CAD software are called CAD mesh models (CMM). The focus of this paper is the CAD mesh model.

Segmentation aims to partition CMM into "meaningful" regions [1]. Each region can be fitted to a distinct, mathematically analyzable form [2]. Literature reveals the 
availability of many mesh segmentation algorithms. However, most of them are not suitable for CMM. Several mesh segmentation approaches in the literature have relied on information such as curvature or sharp edges. Huge time is needed for curvature computation. The curvature is sensitive to noise, variations in dimensions and unevenly distributed triangulations [2]. Several mesh segmentation methods set local threshold while computing curvature. It is difficult to establish a single global threshold [36].

Major research work has been carried out in extracting volumetric and free-form features in the last two decades. However, most feature recognition (FR) tools work on B-rep models. Innovative 3D design and manufacturing methods are mesh based $[7,8]$. A need exists to develop FR from the mesh model. STL format is globally supported by all CAD/CAM system which makes STL a platform-independent data exchange [9]. If we recognize features from STL model, it will be a unique data translator utility $[10,11]$.

The above observations inspire the research work reported in this paper. This paper adopts a hybrid mesh segmentation approach for detecting volumetric features. The algorithm segments the CMM into basic primitives like plane, cylinder, cone, sphere etc. After extraction of analytical surfaces, a rule-based approach is used for feature detections. The innovation lies in the intersecting feature detection in which no tedious curvature information and edge detection technique has been used. We focus on, comparing the hybrid mesh segmentation algorithm with existing and recent state-ofthe-art approaches like Attene et al.[12], Li et al.[13], Yan et al.[1], Adhikary N and Gurumoorthy, B. [14], Le and Duan [15] and RANSAC [16].

The main contributions of this research can be summarized as follows:

- Intelligent threshold prediction makes hybrid mesh segmentation automatic.

- Complex holes lying on multiple planer regions are detected and separated successfully.

- No curvature information has been used for feature detection.

- Feature extracted without edge detection techniques.

- Partitioning criteria used for clustering triangles is "Facet Area".

- Intersecting features are extracted automatically and their parameters are estimated accurately.

The rest of the paper is structured as follows: Section 2 provides a comprehensive review of relevant literature, Section 3 illustrates a proposed methodology for the volumetric feature recognition. Section 4 deals with volumetric feature recognition. Discussion based on results are provided in Section 5. Section 6 present conclusion and future scope.

\section{$2 \quad$ Literature Review}

A comprehensive review of various FR approach with their strengths and weaknesses are reviewed in the literature [8,11,17-21]. As we focus on, comparing robustness and consistency of hybrid mesh segmentation algorithm with existing and recent state-of-the-art approaches, here, we limit our review of to those approaches only. 
Schnabel et al. [16] designed RANSAC (RANdom Sample Consensus) based framework for recognizing basic primitives. However, this approach over or under segment the model results in inaccuracy in feature extraction. Li et al. [13] invented the globFit method which is a modified version of Schnabel et al. [16] approach. This approach is primitive fitting based rather than segmentation. They used parallel, orthogonal equal angle relations in extracted primitives. This approach is computationally costlier and heavily dependent on RANSAC [16] output. Yan et al. [1], presented an algorithm for mesh segmentation of scanned or STL CAD model into non-overlapping patches by fitting quadric surfaces. Each patch was fitted to a general quadrics surface. Criteria used for segmentation was geometric distance based error function. However, the method is suitable for quadric surface only. Not suitable to identify tori or blends.

Muraleedharan et al. [22] used a random cutting plane combined with graph traversal, and Gauss map to extract the interacting features. Their algorithm is unable to separate the interacting features. Fig.1(c) shows the limitation of their approach. They used Gaussian curvature for boundary extraction and separating the interacting features. Their algorithm depends on a number of planes for features extraction which is assumed to be known. The feature must have the presence of inner rings which is the major limitation of the algorithm. If a feature does not have inner rings, it will not be detected. Fig. 1(c) and Fig. 2(d) shows few examples of extracted volumetric features but unable to separate into individual features. Segmentation was not able to separate them as the joints between them have a complex boundary. However, our algorithm detects intersecting features along with geometric parameters.

Adhikary and Gurumoorthy [14] presented an algorithm to recognize free-form volumetric features without segmentation from CMM. They used 2D slicing to identify feature boundaries. Features are identified by extracting feature boundary edges using $3 \mathrm{D}$ seed information of those $2 \mathrm{D}$ features. Region growing technique is used to find features using 3D seed vertex and feature boundary edges. The algorithm does not depend on mesh geometrical properties and mesh triangle density. However, the algorithm is unable to detect and extract parameters of volumetric features for test case shown in Fig. 2 (a). Their algorithm depends on the choice of Minimum Feature Dimension (MFD) and must be known in advance before feature extraction. Fig.2 (d). shows failure case of Adhikary and Gurumoorthy [14].

M. Attene. et al. [12] designed Hierarchical Fitting Primitives (HFP) technique of mesh segmentation which needs visual inspection along with a number of clusters as an input parameter to perform the segmentation. However, knowing a number of clusters before feature extraction is difficult. Fig.1 (b) and Fig.2 (b) shows the failure case of M. Attene et al. [12].

Le and Duan [15] used uniform slicing along the major direction. They used a dimensional reduction technique which transforms $3 \mathrm{D}$ primitives to $2 \mathrm{D}$ to get a profile curve. The primitives are detected based on profile curve analysis. However, the algorithm is slice thickness dependent, and slicing techniques fail to detect or separate complex interacting features as noted by [14]. 
The proposed technique automatically extracts volumetric features like blends and holes along with their geometric parameters. With hybrid mesh segmentation, we can separate the interacting features as well. Fig. 1(d) and Fig.2(e) shows examples of volumetric feature recognition. Hybrid mesh segmentation extracted all the features whereas the closest one among others is the Le and Duan [15].

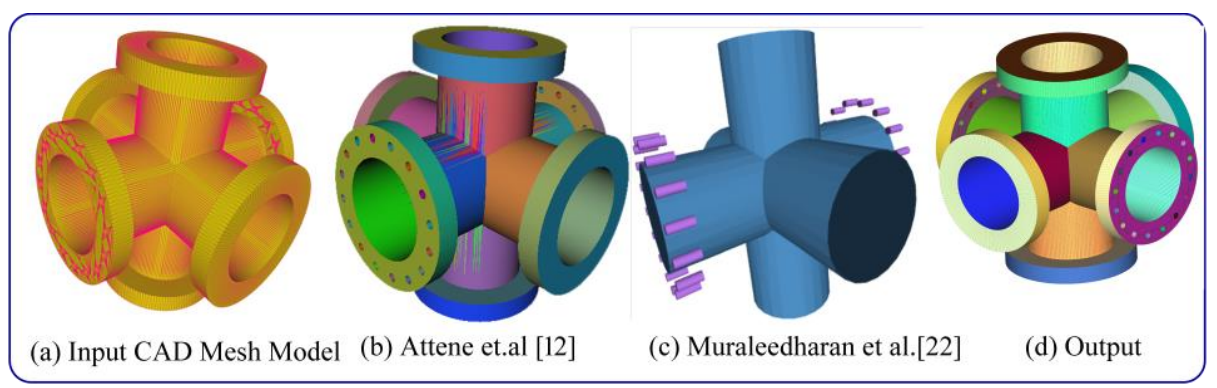

Fig. 1. Failure cases for intersecting volumetric feature

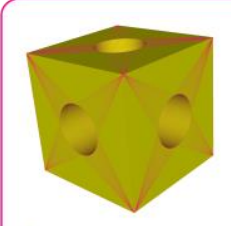

(a) Input $\mathrm{CAD}$ Mesh Model

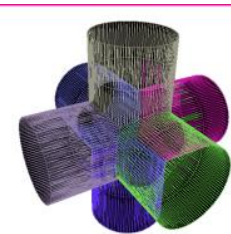

(b) Attene et al. [12] Year : 2006

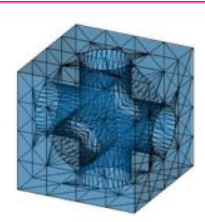

(c) Adhikary et al.[14] (d) Muraleedharan et al.[22] Year : 2016

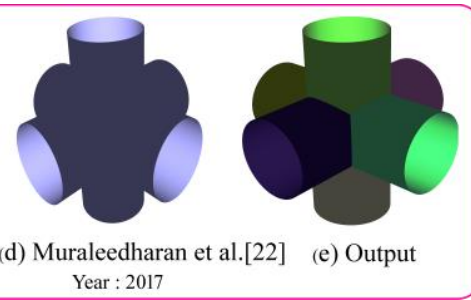
Year : 2017

Fig. 2. Failure case of Muraleedharan et al. [22], Adhikary et al.[14] and Attene et.al [12]

\section{$3 \quad$ Methodology}

The proposed algorithm involves three steps viz. preprocessing, hybrid mesh segmentation and compound hole recognition. Fig. 3 illustrates the overall strategy to extract complex intersecting holes from CMM including hole chain interaction which consists of following steps:

\subsection{Preprocessing}

In preprocessing, we build topology in inputted CAD mesh model.The objective of topology construction is to create and depict the faceted data in an appropriate data structure.

\section{Input CAD Mesh Model}

The proposed method takes a valid CMM as input in ASCII or Binary format. In this research work, we assume a valid STL model as an input which is free from errors, hence not requiring model healing [11].

\section{Automatic Threshold Prediction}

The facets laying on the same surface have the same quality. We use the "Facet Area" property to segment the model. A significant step in segmentation is to set the appro- 
priate ADF (threshold) at the beginning. It is a cumbersome task of identifying a threshold value for getting the expected results. Most of the time a trial and error approach is used to identify a correct threshold [22]. Inadequate threshold leads to oversegmentation (multiple small patches) or under segmentation. Over-segmentation needs a post-processing merging step and increase of processing time whereas under segmentation leads to deficient results[23]. However, for a layman, setting the appropriate threshold is too complicated. Manual prediction is laborious and errors prone. Therefore, an automatic and intelligent prediction approach is of significance [24].

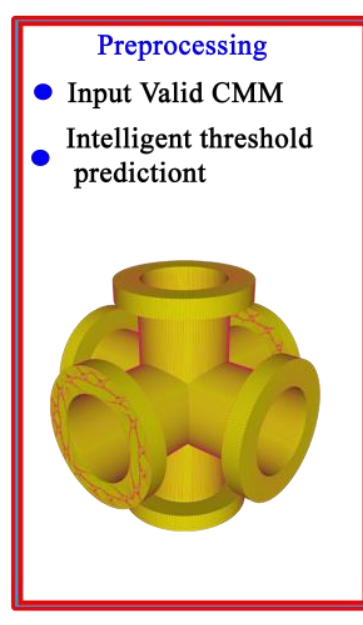

(a) Preprocessing

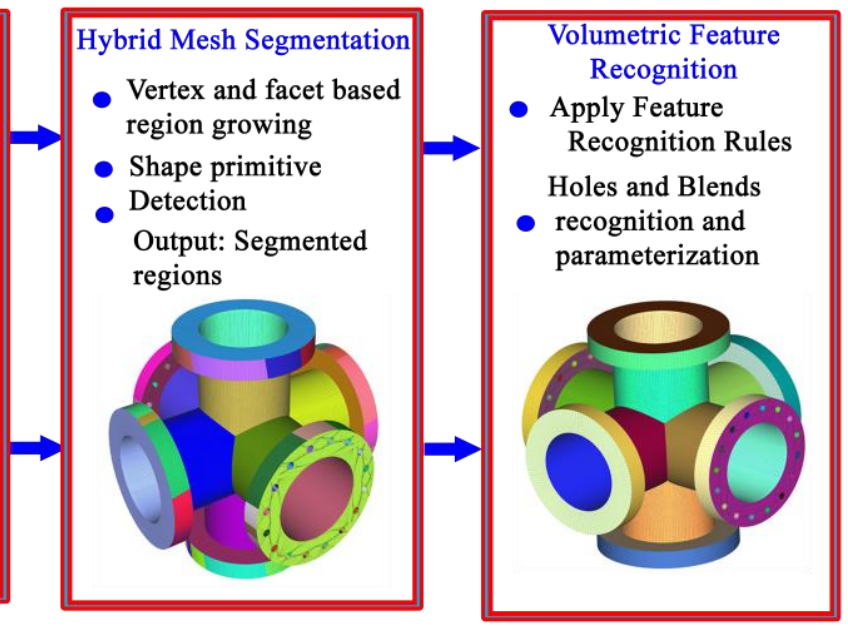

(c) Volumetric Feature Recognition

Fig. 3. The framework of the proposed methodology

As stated above, Area Deviation Factor (ADF) is the decisive factor in segmentation quality. We have proposed and implemented intelligent prediction of threshold using a machine learning classifier to partition CMM. We have performed supervised machine learning for the prediction of Area Deviation Factor (Threshold) automatically. A detailed description of automating threshold prediction using machine learning classifier is beyond the scope of this paper.

\subsection{Hybrid mesh segmentation}

The goal of hybrid mesh segmentation is to partition CMM into basic primitives like a plane, sphere, cylinder, and cone. It is difficult to segment CMM by using facet based region growing or vertex based region growing alone. Vertex-based region growing technique is used to detect curved surface whereas Facet-based growing technique is used to detect curved features and planes. None of these techniques on their own gives a robust solution to recognize feature from CMM.

Hybrid mesh segmentation uses region growing algorithms to clusters facets into groups. Figure 4 illustrates the structure of the proposed hybrid mesh segmentation methodology. The approach is hybrid as we use the "Facet Area" property to group facets together, using a combination of vertex-based and facet-based region growing 
algorithms [25]. After segmentation, each facet group is subjected to several conformal tests, to identify the type of analytical surface it might be representing such as a cylinder, cone, sphere etc

A promising approach that has become evident is a hybrid (facet and vertex based) one wherein the advantages of the above approaches are combined A detailed description of Hybrid mesh segmentation is beyond the scope of this paper.

After extraction of analytical surfaces, feature boundaries are identified in analytical surfaces.

\subsection{Iterative region Merging}

The Hybrid mesh segmentation leads to over-segmentation. The over segmented regions are need to be merged again to generate the single region. The proposed iterative region merging technique is based on predefined merging criteria. The iterative region merging technique repeatedly merged regions that have similar geometric property. Following steps has been carried out in iterative region merging.

\section{Region Merging}

Region merging merge features iteratively. A single pass is not enough to merge all features. Only if two features are adjacent, they will be merged to one if they satisfy geometry equality test. After merging, adjacency may have changed, so features that were not eligible for merging in the previous pass will be merged in next pass.

\section{Reclamation}

After region merging, small cracks are observed close to the corner and at the region boundaries [26]. To make a watertight model, these uncollected facets are reclaimed into the surrounding identified regions (Feature) based on reclamation criteria.

Fig. 4 shows examples of the cylindrical region generated by the hybrid mesh segmentation, Fig. 3(a) shows the original mesh models, Fig. 4(a) demonstrates the segmentation results (12 planes and 523 cylindrical patches), Fig. 4(b) demonstrates the region is merging results, Fig. 4(c) demonstrates the reclamation results, and Fig. 4(d) illustrates the final region merging after reclamation(12 planes and 50 cylinders). The system takes approximately 1.759 seconds for feature detection

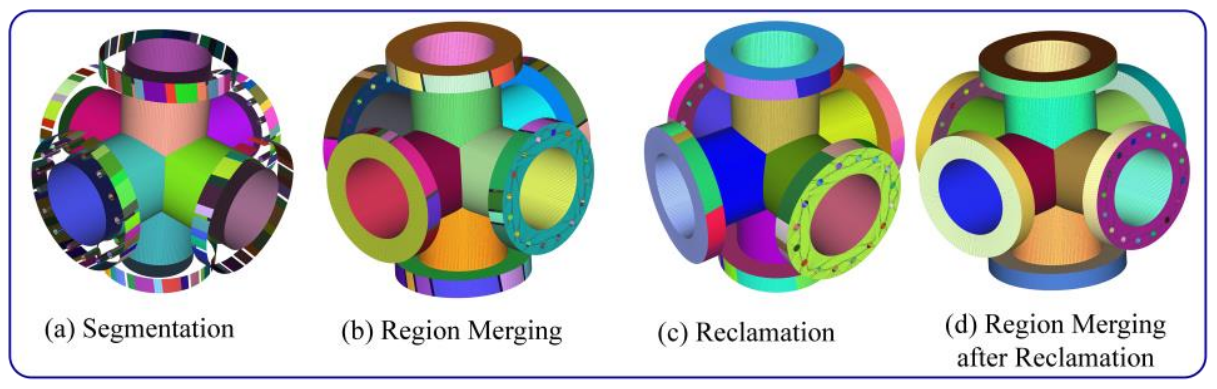

Fig. 4. Hybrid mesh segmentation process

\section{Volumetric feature recognition}

We detect volumetric features like holes and blends by applying a set of rules based on adjacency information of the primitives detected in the previous step. Most of the 
existing approaches evaluate pockets, slots and hole interaction. However, $60 \%$ of the average portion of the total facets in CAD mesh model are of blends features [27], and holes constitute a significant percentage of features in mechanical engineering parts. Hence, we consider blends and hole recognition.

To test the algorithms ability to recognize volumetric features, test cases have been prepared. Using random color for different primitives, features can be interpreted.

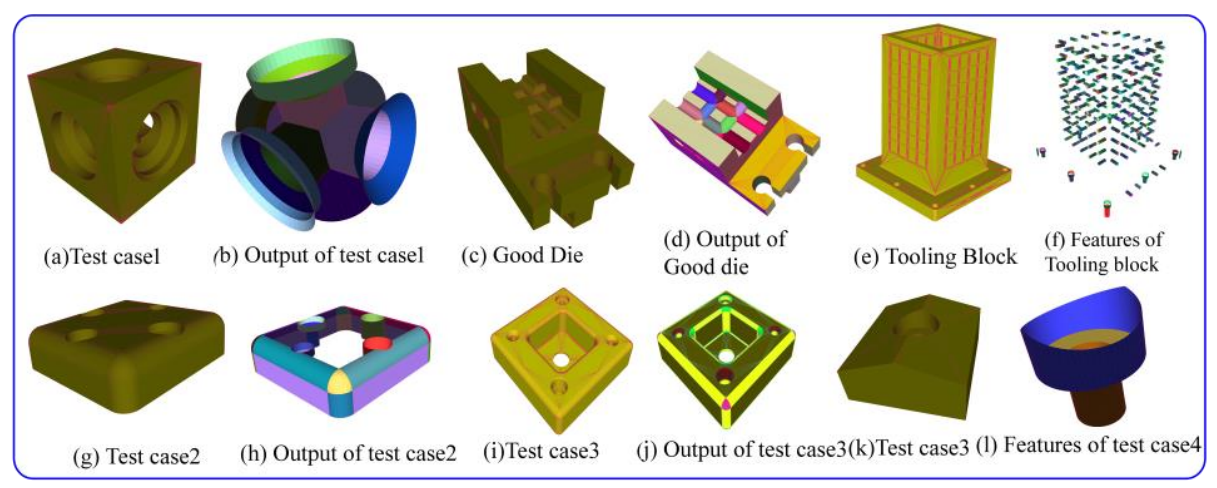

Fig. 5. illustrates the interacting feature recognition of a model

Table 1 summarizes the performance measure for a proposed algorithm for the test cases shown in Figures 5a,5c,5e, 5g, 51 and 5k. We use percentage coverage as an indicator of the successful segmentation algorithm. It is a ratio of a number of features recognized to the number of features present in a CAD mesh model.

Table 1. A quantitative comparison of CAD mesh Model

\begin{tabular}{|c|c|c|c|c|c|c|r|r|}
\hline Test Cas es & $\mathbf{F}$ & $\mathbf{V}$ & $\mathbf{S}$ & $\mathbf{A d f}$ & $\mathbf{N}_{\mathbf{R b r m}}$ & $\mathbf{N}_{\text {Rarm }}$ & $\mathbf{T}$ & $\mathbf{C}$ \\
\hline Figure 5a & 1640 & 812 & 0.417 & 0.8 & 39 & 20 & 0.211 & 100 \\
\hline Figure 5c & 2472 & 1230 & 0.624 & 0.6 & 55 & 29 & 0.864 & 99.67 \\
\hline Figure 5e & 38932 & 19092 & 9.84 & 0.7 & 1169 & 630 & 4.257 & 99.58 \\
\hline Figure 5g & 1380 & 690 & 0.349 & 0.75 & 36 & 25 & 0.254 & 99.28 \\
\hline Figure 5i & 12068 & 6034 & 2.23 & 0.75 & 158 & 69 & 1.078 & 100 \\
\hline
\end{tabular}

F : Number of Facets $\quad$ V : Number of Vertex $\quad$ S : STL Size (in MB)

Adf : Predicted Area deviation factor $\quad \mathrm{C}: \%$ Coverage

NRbrm : Number of regions before region merging

NRarm : Number of regions after region merging $\mathrm{T}$ : Overall Timing (in a second)

5 Results and discussion

\subsection{Comparison with recently developed algorithm}

The comparison was done with existing state-of-the-art approaches like RANSAC [16], Attene et.al.[12], Li et al. [13] where code publicly available. The results for Le and Duan [15] are taken from [15] as the code was not available. The proposed approach does not depend on attributes like curvature, minimum feature dimension, 
number of clusters, number of cutting planes, the orientation of model and thickness of the slice to extract volumetric features.

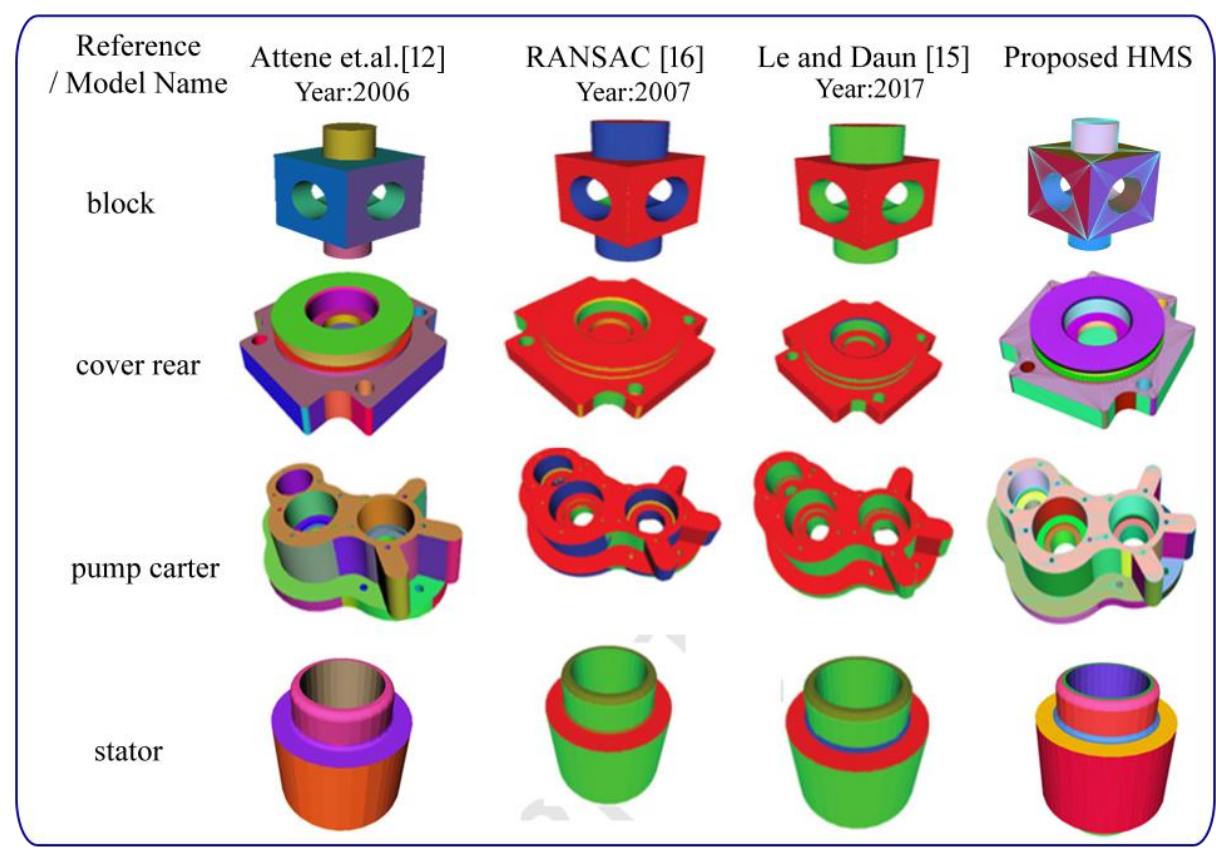

Fig. 6. : Comparison with the existing algorithm

Table 2 summarizes the quantitative comparison for a proposed algorithm for the test cases. We evaluate using number of primitives, the coverage percentage, the distance error. As noted in Fig.6, the proposed algorithm yields better results than RANSAC [16] and Attene et.al.[12]. Our results are comparable to Le and Duan [15].

Table 2. Quantitative evaluation of primitive quality in Figure 6

\begin{tabular}{|c|c|c|c|c|c|c|c|c|c|c|c|c|c|c|c|}
\hline \multirow{2}{*}{$\begin{array}{l}\text { Model } \\
\text { Name }\end{array}$} & \multicolumn{5}{|c|}{ \# of Primitives } & \multicolumn{5}{|c|}{ Coverage (\%) } & \multicolumn{5}{|c|}{ Distance Error $\left(\times \mathbf{1 0}^{-3}\right)$} \\
\hline & $\mathbf{I}$ & II & III & IV & $\mathbf{V}$ & $\mathbf{I}$ & II & III & IV & $\mathbf{V}$ & $\mathbf{I}$ & II & III & IV & $\mathbf{V}$ \\
\hline Block & 14 & 14 & 14 & 9 & 14 & 100 & 100 & 100 & 64.3 & 99 & 0.04 & 0.37 & 0.08 & $\mathrm{n} / \mathrm{a}$ & 0.69 \\
\hline Cover rear & 45 & 28 & 45 & 45 & 28 & 100 & 87.8 & 100 & 100 & 87.8 & 0.02 & 0.11 & 0.04 & $\mathrm{n} / \mathrm{a}$ & 0.15 \\
\hline Pump carter & 83 & 57 & 63 & 76 & 57 & 99.5 & 92.9 & 98.6 & 99.2 & 92.9 & $\mathbf{0 . 0 3}$ & 0.16 & 0.3 & $\mathrm{n} / \mathrm{a}$ & 2.3 \\
\hline Stator & 12 & 12 & 12 & 6 & $\mathrm{n} / \mathrm{a}$ & 100 & 100 & 100 & 50 & $\mathrm{n} / \mathrm{a}$ & 0.01 & 0.8 & 0.47 & $\mathrm{n} / \mathrm{a}$ & $\mathrm{n} / \mathrm{a}$ \\
\hline
\end{tabular}

*(I) Proposed algorithm (II) RANSAC [16] (III) Le and Daun [15] (IV) GlobFit et.al.[12] (IV) 


\section{Conclusion}

In this research, an elegant method has been proposed and implemented for volumetric features from CMM using a hybrid region growing approach. We have used the rule-based approach for feature recognition. The proposed algorithm captures and separates intersecting features.

Comparing with existing recent approaches such as Muraleedharan et al. [22], Adhikary et al. [14], Attene et al. [12], RANSAC [16], Le and Daun [15] and other on benchmark test cases, the proposed technique successfully recognized the features such as blends, compound holes and their interactions and found to be robust and consistent with coverage of more than $95 \%$ in addressing volumetric feature. The proposed approach is simple, more general and more reliable.

In the future, we plan to extend our algorithm to capture the parent-child relationship in detected features.

\section{Acknowledgments}

This work has been supported by Centre for Computational Technologies (CCTech), Pune, India. We also appreciate the authors of the RANSAC [16] and Attene et.al.[12]. GlobFit et.al.[12] for making their code publicly available. Authors are also grateful to Dr. Truc Le and Dr.Ye Duan for helping us to compute coverage (\%).

\section{References}

1. D.-M. Yan, W. Wang, Y. Liu, and Z. Yang.: Variational mesh segmentation via quadric surface fitting. Computer-Aided Design 44(11), 1072-1082, (2012).

2. S. Xú, N. Anwer, C. Mehdi-Souzani, R. Harik, and L. Qiao.: STEP-NC based reverse engineering of in-process model of NC simulation. The International Journal of Advanced Manufacturing Technology 86(9-12), 3267-3288, (2016).

3. T. Várady, M. A. Facello, and Z. Terék.: Automatic extraction of surface structures in digital shape reconstruction. Computer-Aided Design 39(5), 379-388 (2007).

4. P. Benkö and T. Várady.: Segmentation methods for smooth point regions of conventional engineering objects. Computer-Aided Design 36(6), 511-523 (2004).

5. P. Csákány and A. M. Wallace.: Computation of Local Differential Parameters on Irregular Meshes. In The Mathematics of Surfaces IX, R. Cipolla and R. Martin, Eds. London: pp. 19-33. Springer London, (2000).

6. J. Huang and C. H. Menq.: Automatic data segmentation for geometric feature extraction from unorganized 3-D coordinate points. IEEE Transactions on Robotics and Automation 17(3), 268-279 (2001).

7. D.-B. Tang, L. Zheng, and Z. Li.: An Intelligent Feature-Based Design for Stamping System. The International Journal of Advanced Manufacturing Technology 18(3), 193200(2001).

8. J. Corney, C. Hayes, V. Sundararajan, and P. Wright.: The CAD/CAM Interface: A 25Year Retrospective. Journal of Computing and Information Science in Engineering 5(3), 188-197 (2005). 
9. M. T. Hayasi and B. Asiabanpour.: Extraction of manufacturing information from designby-feature solid model through feature recognition. The International Journal of Advanced Manufacturing Technology 44(11-12), 1191-1203 (2009).

10. F. Bianconi.: Bridging the gap between CAD and CAE using STL files. International Journal of CAD/CAM 2(1), 55-67(2002).

11. V. B. Sunil and S. S. Pande.: Automatic recognition of features from freeform surface CAD models. Computer-Aided Design 40(4), 502-517 (2008).

12. M. Attene, B. Falcidieno, and M. Spagnuolo.: Hierarchical mesh segmentation based on fitting primitives. The Visual Computer 22(3), 181-193 (2006).

13. Y. Li, X. Wu, Y. Chrysathou, A. Sharf, D. Cohen-Or, and N. J. Mitra.: GlobFit: Consistently Fitting Primitives by Discovering Global Relations. In ACM SIGGRAPH 2011 papers on - SIGGRAPH '11, 2011, vol. 30, no. 4, pp. 52.ACM (2011).

14. N. Adhikary and B. Gurumoorthy.:A slice based approach to recognize and extract freeform volumetric features in a CAD mesh model. Computer-Aided Design and Applications13(5), 587-599 (2016).

15. T. Le and Y. Duan.: A primitive-based 3D segmentation algorithm for mechanical CAD models. Computer Aided Geometric Design 52-53, 231-246 (2017).

16. R. Schnabel, R. Wahl, and R. Klein.: Efficient RANSAC for Point-Cloud Shape Detection. Computer Graphics Forum 26(2), 214-226 (2007).

17. J. J. Shah, D. Anderson, Y. S. Kim, and S. Joshi.:A discourse on geometric feature recognition from CAD models. Journal of computing and information science in engineering 1(1), 41-51 (2001).

18. B. Babic, N. Nesic, and Z. Miljkovic.: A review of automated feature recognition with rule-based pattern recognition. Computers in Industry 59(4), 321-337(2008).

19. A. K. Verma and S. Rajotia.: A review of machining feature recognition methodologies. International Journal of Computer Integrated Manufacturing 23(4), 353-368 (2010).

20. R. Zbiciak and C. Grabowik.: Feature Recognition Methods Review. in International Conference on Renewable Energy Sources-Research and Business, vol. Proceeding, E. Rusiński and D. Pietrusiak, Eds. Cham: Springer International Publishing, 2017, pp. 605615. Springer, (2017).

21. D. Xiao, H. Lin, C. Xian, and S. Gao.: CAD mesh model segmentation by clustering. Computers \& Graphics 35(3), 685-691(2011).

22. L. P. Muraleedharan, S. S. Kannan, A. Karve, and R. Muthuganapathy.:Random cutting plane approach for identifying volumetric features in a CAD mesh model. Computers \& Graphics 70, 51-61(2018).

23. A. Agathos, I. Pratikakis, S. Perantonis, N. Sapidis, and P. Azariadis.:3D Mesh Segmentation Methodologies for CAD applications. Computer-Aided Design and Applications 4(6), 827-841(2007).

24. F. Qin, L.-Y. Li, S.-M. Gao, X.-L. Yang, and X. Chen.:A deep learning approach to the classification of 3D CAD models. Journal of Zhejiang University SCIENCE C, 15(2), 91106 (2014).

25. V. Hase, S. Verma, Y. Bhalerao, S. Jadhav, and G. Vikhe Patil.: Manuscripts submitted for publication, Unpublished work.

26. H. S. Kim, H. K. Choi, and K. H. Lee.: Feature detection of triangular meshes based on tensor voting theory. Computer-Aided Design 41(1), 47-58 (2009).

27. N. Rafibakhsh and M. I. Campbell.: Hierarchical Fuzzy Primitive Surface Classification From Tessellated Solids for Defining Part-to-Part Removal Directions. Journal of Computing and Information Science in Engineering 18(1), 011006 (2017). 